\title{
Influence of plasma modification on mechanical and thermal properties of Polypropylene/ Nano- Calcium Silicate Composites
}

\author{
Ajeesh. $G^{1 *}$, Aditya Raman ${ }^{1}$, Adarsh Parameswaran ${ }^{1}$, Sundhara Pandiyan ${ }^{1}$, Parvathy ${ }^{1}{ }^{1}$ \\ and Philip George ${ }^{2}$ \\ ${ }^{1}$ Department of Aerospace Engineering, Amrita School of Engineering, Amrita Vishwa \\ Vidyapeetham, Coimbatore-641112, India \\ ${ }^{2}$ Vijnan Institute of Science and Technology, Elanji, Ernakula, Kerala, India
}

\begin{abstract}
The aim of the research is to study the influence of plasma modification on nano calcium silicate/polypropylene composites. Polypropylene (PP) is considered for this study as it possesses high impact strength, toughness and availability. Calcium silicate is considered as reinforcement because of its high temperature resistance, high flexural strength and high strength to mass ratio. Fourier transform infrared spectroscopy (FTIR) results show that there is a change in the functional group on the surface of calcium silicate after modification. ThermoGravimetric Analysis (TGA), Differential Scanning Calorimetry (DSC) results show that the decomposition temperature increased with increasing amount of filler particles. It is also observed that the modification has produced a marginal increase in the decomposition and glass transition temperature. Tensile test results also show a gradual increase in the tensile properties of composites when high ratio is reinforcement. Tensile test results also show that there is a marginal increase in the tensile strength when reinforced with modified calcium silicate when compared to nonmodified. Scanning Electron Microscopy (SEM) reveals that there is a enhanced dispersion of nano particles on modification. Based on the findings it can be concluded that plasma modification enhances the thermal and mechanical property marginally.
\end{abstract}

Keywords: Polymeric nano composites, Plasma modification, Scanning Electron Microscopy, Differential scanning calorimeter, Superior dispersion.

\section{Introduction}

From the start of human existence, materials have played a significant role in the development of civilization. In the late $19^{\text {th }}$ century Composites gained importance because of its high strength, low weight, superior thermal properties and design flexibility. The first composite made by man was having mud as matrix and straw as reinforcement. Among metal matrix, ceramic matrix and polymer matrix composites, polymer composites have a

\footnotetext{
* Corresponding author: g_ajeesh@.cb.amrita.edu
} 
high resistance to chemical corrosion and scratching. They provide easy fabrication of very complex parts with low tooling cost. Thermoplastic polymers are chosen over thermoset due to their advantages such as high toughness, shorter manufacturing cycles, reprocessing possibilities and they also do not require refrigeration storage. Carbon short fiber or glass short fiber reinforced in thermoplastic matrices such as polyetherimide (PEI), polyetheretherketone (PEEK) and polyphenylene sulfide (PPS) have been studied considering their excellent mechanical and thermal properties. The major drawback of these polymers are that they are costly and difficult to process when compared to thermosetting composites. Considering the cost and ease of manufacturing, polypropylene (PP) was considered as matrix for this research [1]. Polypropylene is a semi-crystalline thermoplastic and is known for its superior thermal and chemical properties.

Polypropylene can be used in automobiles appliances in which creep resistance needs to be considered in addition to stiffness and toughness. But non reinforced PP resin has low mechanical and thermal properties. To improve its strength and thermal properties, various types of fillers (such as fibres, clay, silica, graphene and CNT) have been incorporated. Calcium silicate is considered for this study as it possesses high thermal properties. Research has been performed on modification of nano particles and it has been noted that modification of nano particles enhances the mechanical and thermal properties of the composites. It is also observed that the filler show better dispersion on modification $[2,3]$. The modification of nano calcium silicate has not been performed in the past and this research would help in understanding the influence of plasma modification on nano calcium silicate- polymer composites.

\section{Experimental}

\subsection{Materials}

Isotactic polypropylene of grade H110MA supplied by Repol, India was used. Nanocalcium silicate was procured from Sigma Aldrich, Switzerland.

\subsection{Plasma modification}

Plasma treatment is a surface modification technique that uses the fourth state of matter plasma. In order to attain the plasma state, high energy is provided to a gas $\left[\mathrm{Ar}, \mathrm{O}_{2}, \mathrm{~N}_{2}\right.$, etc] which ionizes it. The process is carried out under vacuum conditions. Oxygen plasma removes organic contaminants by reacting chemically with highly reactive oxygen radicals. Oxygen plasma surface treatment promotes surface oxidation and hydroxylation $(\mathrm{O}-\mathrm{H}$ groups) which increases the surface wettability. The figure of plasma modification of nano calcium silicate is as shown in the Fig. 1. 
Fig. 1. Plasma Modification of nano-calcium silicate

\subsection{Preparation of PPI (unmodified/ modified) nano calcium silicate composites}

The compounding was done using twin-screw extruder. The compounded mixture was then fed into injection molding for preparation of the specimens. Weight percentage of $0.5,1$ and 1.5 were the mass ratios taken initially for both modified and unmodified calcium silicate. The extruder screw rpm was 71 and the die temperature was $232^{\circ} \mathrm{C}$. The outcome was in the form of strands which was then cut to smaller pieces using a shear cutter and then fed into the injection molding machine. The mold temperature was set as $250^{\circ} \mathrm{C}$. Polypropylene containing more than 5\% filler had to be manufactured using Twin screw extruder. These pieces were then injection molded to obtain the specimens.

\section{Characterization}

\subsection{FTIR spectroscopy}

The effect of modification in the nano filler was observed using a Nicolet iS10 machine manufactured by Thermo Scientific, USA. Few grams of the filler was dried and grounded along with $200 \mathrm{mg}$ of $\mathrm{KBr}$. They were then made into transparent pellets and the interferogram was obtained which was converted into a spectrum using Fourier transform.

\subsection{Tensile test}

Tensile test was done using a Universal Testing Machine. The machine used was Zwick/ Roell Z010. The gauge length of the specimen was $50 \mathrm{~mm}$ and the cross-head speed was $1 \mathrm{~mm} / \mathrm{min}$ according to ASTM D-638.

\subsection{Thermo-gravimetric analysis}

Thermo Gravimetric Analysis is performed to study the influence of fillers on the decomposition temperature of composites. SDT Q600 V20.9 Build 20 instrument 
manufactured by TA Instrument Inc., USA, was used to measure the thermal stability of the sample.

\subsection{Differential scanning Calorimetry}

Differential scanning Calorimetry (DSC) is performed to understand the influence of nano calcium silicate on the glass transition temperature of composites. DSC Q20 V24.10 Build 122 instrument manufactured by TA Instrument Inc., USA, was used. Few grams of samples was measured and kept in the aluminium DSC pan provided and heated up to $300^{\circ} \mathrm{C}$ in nitrogen atmosphere. The heat flow curves were obtained as a function of temperature from which the melting temperature was determined.

\subsection{Scanning Electron Microscopy}

SEM is performed to study the effect of modification on the dispersion of the nano fillers in the composites.

\section{Results and Discussion}

\subsection{FTIR spectroscopy of the unmodified and the plasma modified calcium silicate fillers}

Comparing the FTIR spectrum of the unmodified nano calcium silicate as shown in Fig. 2, and the spectrum of the plasma-modified filler as shown in Fig. 3, it is observed that the transmittance value of the hydroxyl group $(\mathrm{O}-\mathrm{H})$ [corresponding to the wavenumber range $3200-3600$ ] has increased by $18 \%$ (from $50 \%$ to $68 \%$ ); alkene group $(\mathrm{C}=\mathrm{C})$ [corresponding to wavenumber $1620-1680$ ] and aromatic group $(\mathrm{C}=\mathrm{C})$ [corresponding to wavenumber $1400-1600$ ] by $12 \%$ (from $68 \%$ to $80 \%$ ) and another alkene group ( $=\mathrm{C}-\mathrm{H})$ by $14 \%(70 \%$ to $84 \%$ ) for the modified filler. The absorption band around $1640 \mathrm{~cm}^{-1}$ is commonly assigned to $\mathrm{O}-\mathrm{H}$ bending vibration but can occur also from vibrations of the $\mathrm{SiO}_{2}$ network and is often hidden by water $\mathrm{O}-\mathrm{H}$ band.

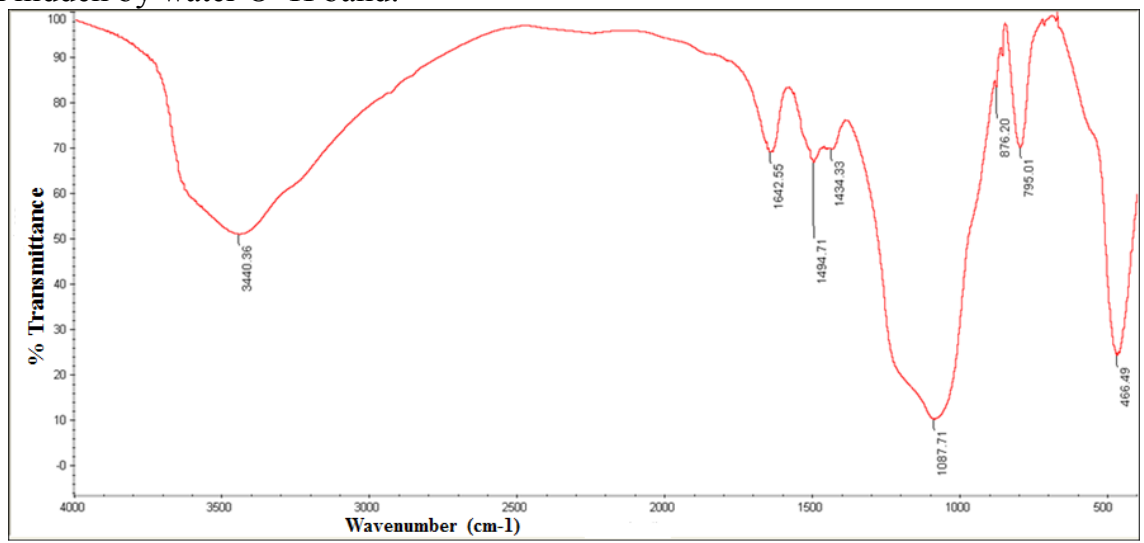

Fig. 2. FTIR plot of untreated Calcium Silicate

The FTIR spectra (Figure 2) reveal an absorption band at $1090 \mathrm{~cm}^{-1}$ due to C-O stretched vibration and a characteristic very narrow band for the spectra are dominated by a broad signal in the $750-1300 \mathrm{~cm}^{-1}$ spectral range, where the stretching vibrations of the $\mathrm{Si}-\mathrm{O}-\mathrm{Si}$ and $\mathrm{Si}-\mathrm{O}-\mathrm{Ca}$ bridges occur [4]. 


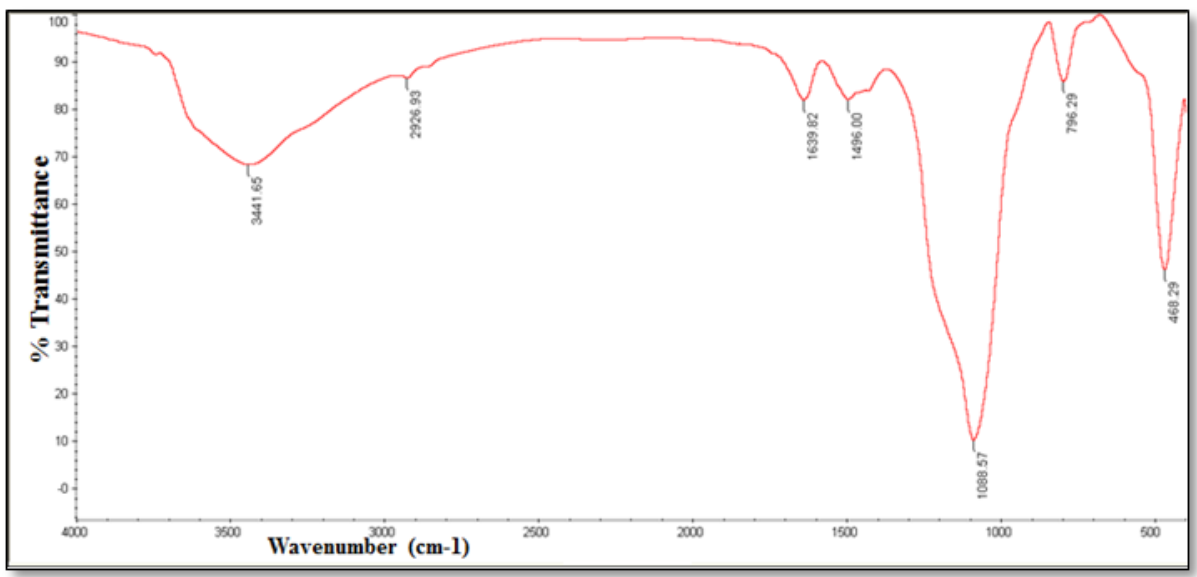

Fig. 3. FTIR plot of treated Calcium Silicate

\subsection{Tensile Tests}

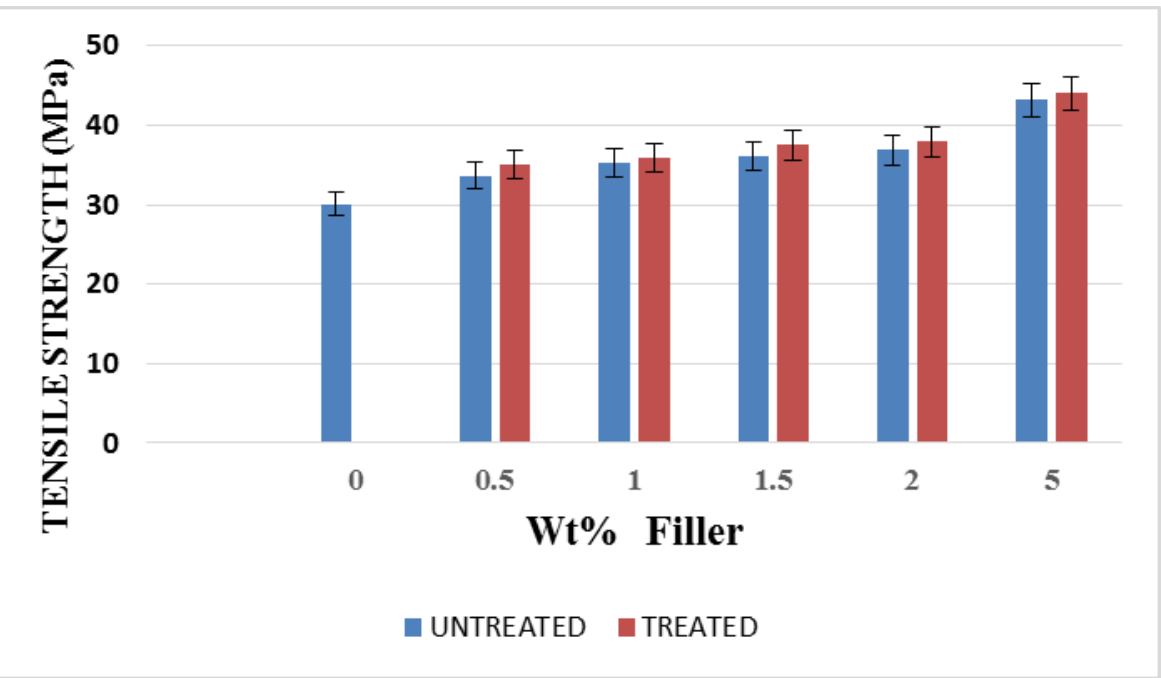

Fig. 4. Tensile strength of composites

It is observed from Fig. 4 and Fig. 5 that the tensile strength and the Young's modulus increases significantly with increasing mass ratio for the composite containing unmodified calcium silicate. The increase is because the filler absorbs energy [5]. The nano filler have higher surface area and hence significant increase is seen. On the contrary it is observed that on modification, there has been a marginal increase compared to untreated nano calcium silicate. This may be attributed to better dispersion. 


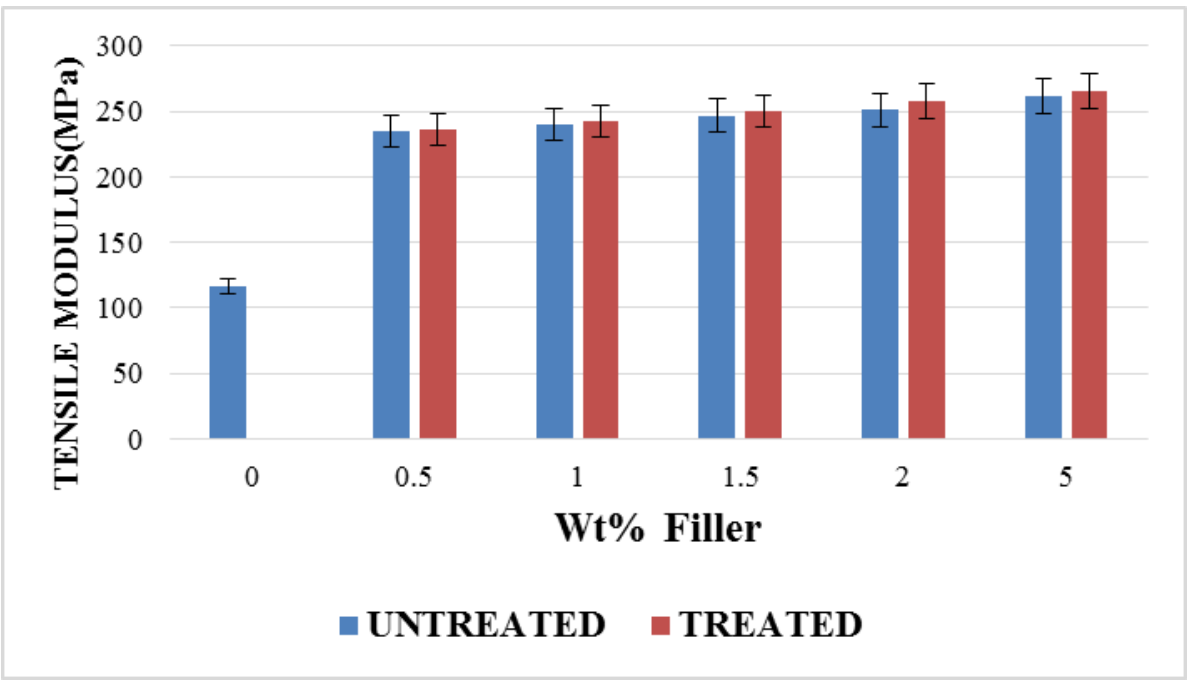

Fig. 5. Tensile modulus of the composites

\subsection{TGA}

It is observed from Fig. 6 that the addition of the fillers increased the decomposition temperature of the composite. Since smaller mass ratios were taken, the increase was not prominent. Both the unmodified and the modified filler showed the same pattern of increase. The thermal stability of the PP matrix increased with the introduction of small amounts of nano calcium silicate. The high specific area of nano calcium silicate also contributed to the greater interaction between the nano filler and the PP matrix which led to the enhancement of both interfacial bonding and thermal stability.

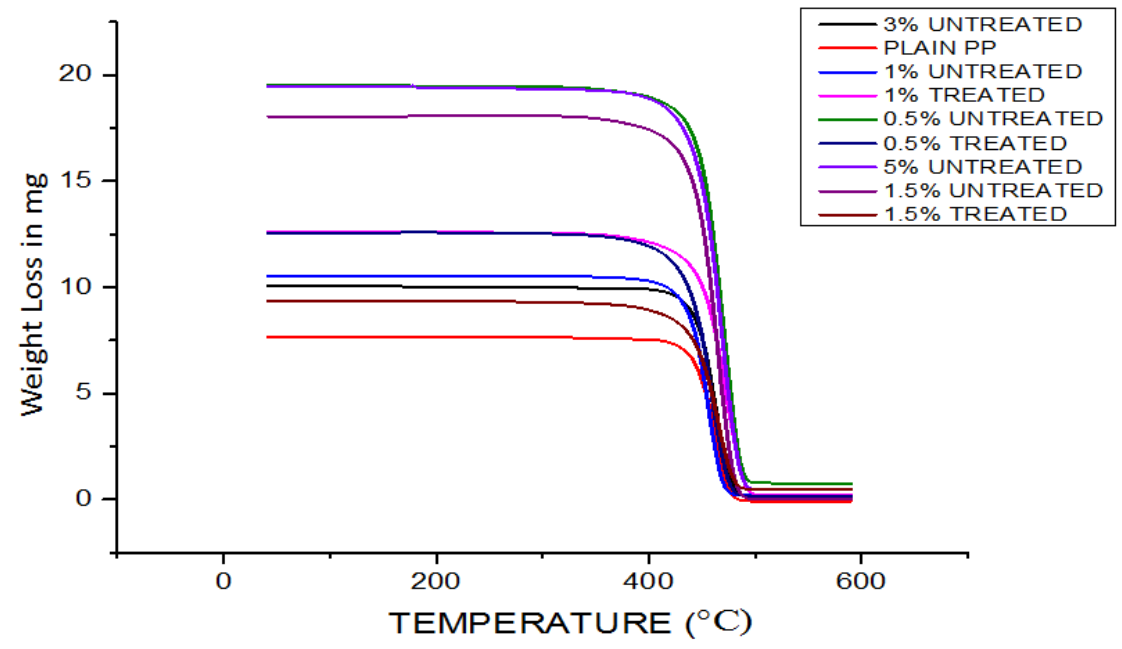

Fig. 6. TGA Plots of composites

This result also indicated that the well-dispersed nano-calcium silicate particles successfully acted as effective nucleation agents in the PP matrix composites. The increase in the thermal stability for both the unmodified and the modified fillers may be attributed to the presence of filler loading. 


\subsection{DSC}

It is observed from the heating curve (Fig. 7) that the melting transition temperature (defined by the maximum at the endothermic peak) is not influenced by the presence of nano-fillers. The slight fluctuations may be due to the impurities in the crucible $[6,7]$. The broadening of the melt endotherm has been associated with the formation of less perfect crystals because of the spatial hindrance of the filler $[8,9]$.

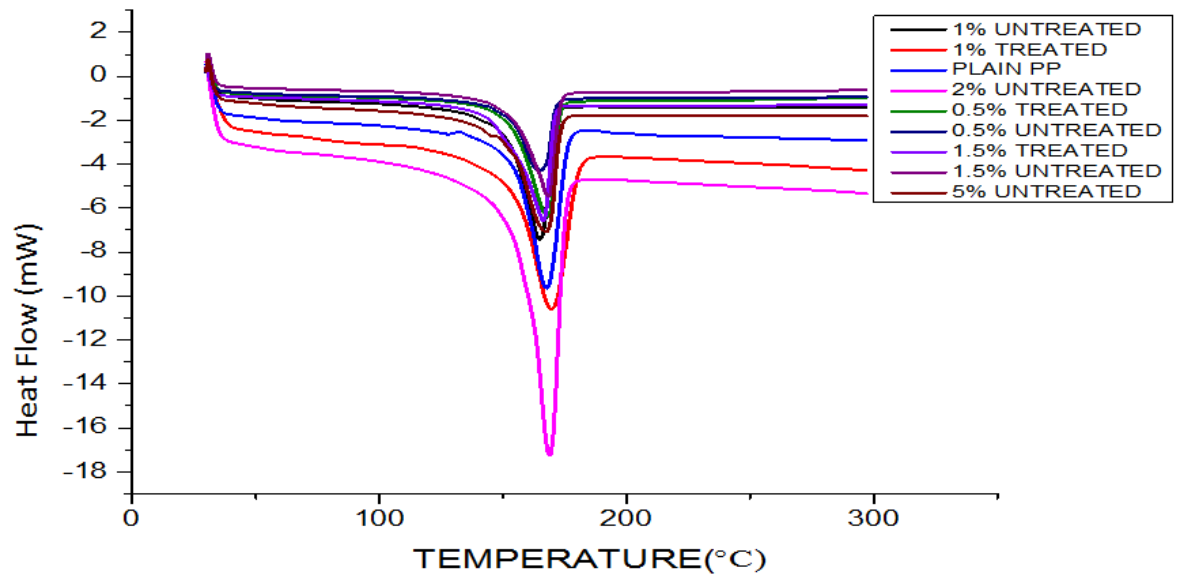

Fig. 7. DSC plot of composites

\subsection{SEM}

SEM was carried out to study the dispersion of nano filler in the composites. Fig. 8 shows the composite figures which does not contain nano filler. Fig. 9 and Fig. 10 shows the composites with untreated and treated nano fillers.

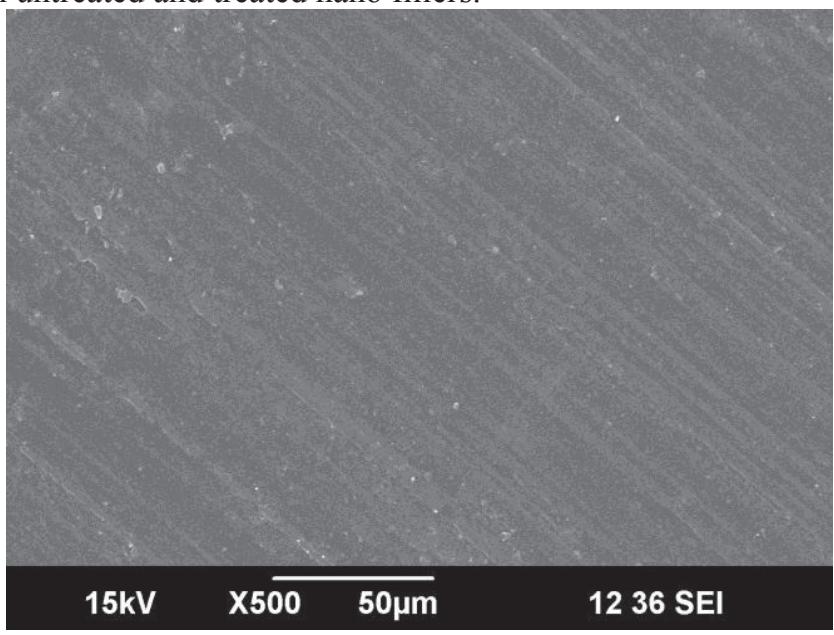

Fig. 8. SEM image of non-reinforced composites 


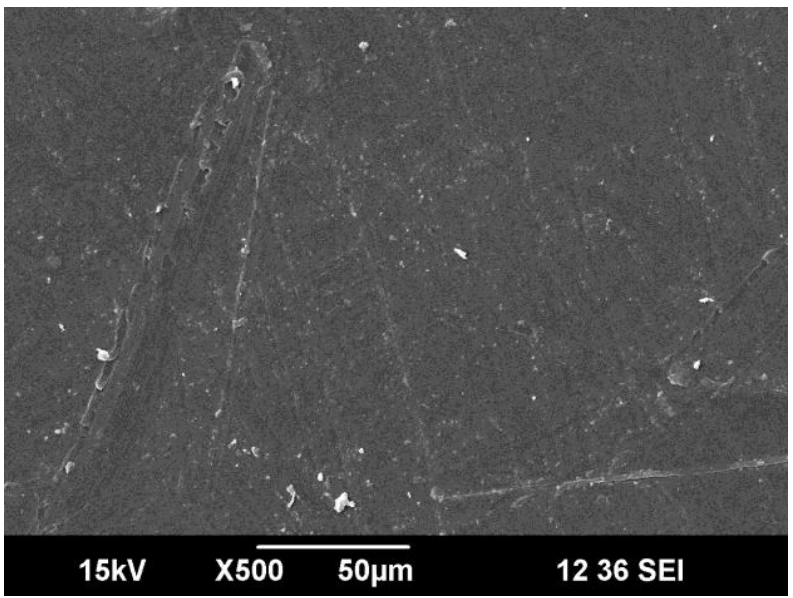

Fig. 9. SEM image of untreated nano filler composites

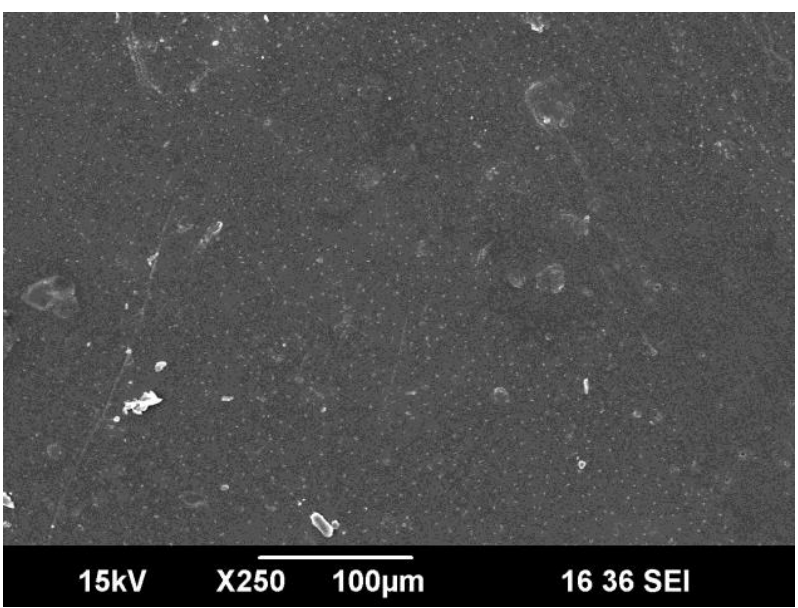

Fig. 10. SEM image of treated nano filler composites

Fig.11 shows a more magnified image of plasma modified calcium silicate composite. This shows dispersion of nano filler within the polymer.

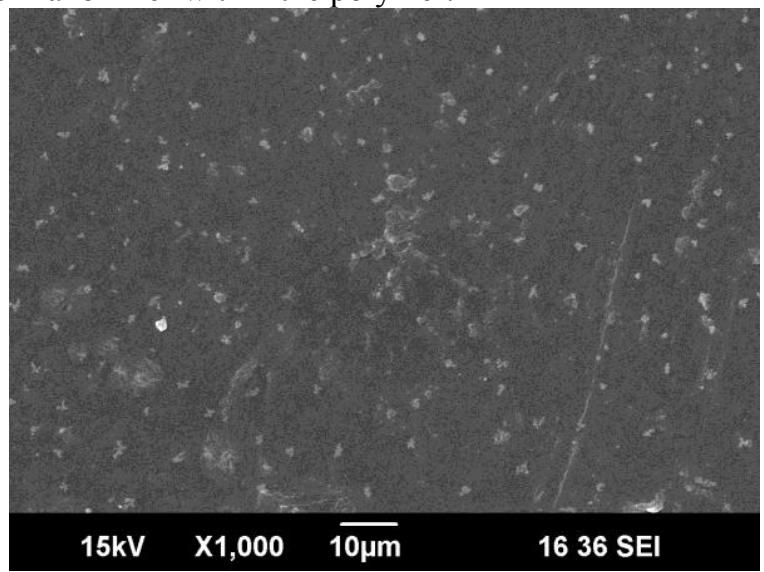

Fig. 11. Magnified SEM image to show enhanced dispersion 
Based on these findings it is evident that plasma modified nano filler shows better dispersion.

\section{Conclusions}

The study revealed the following conclusions:

- The mechanical properties of the composite containing unmodified filler had increased with increasing mass ratio.

- The decomposition temperature of the composite containing unmodified filler increased with increasing mass ratio.

- There was no much change observed in the melting temperature values. But there were marginal fluctuations. This may be because the ratio of filler used was not high.

- The oxygen plasma modification done on the filler increased its surface energy. Hence there was proper bonding between the filler and the polymer. Therefore the composites containing modified calcium silicate gives marginally better results with regard to tensile and dispersion properties.

\section{References}

1. M. Gabr, W. Okumura, H. Ueda, W. Kuriyama, K. Uzawa, I. Kimpara. Composites Part B: Engineering, 69, 94-100 (2015).

2. G. Ajeesh, S. Bhowmik, V. Sivakumar, L. Varshney, Journal of Composite Materials, 51(8), 1057-1072 (2017).

3. G. Ajeesh, Shantanu Bhowmik, Sivakumar Venugopal, Lalit Varshney, Abrar Baluch, Yurim Park, Son Gilsang and Chun Gon Kim. High Performance Polymers, 28(5), 570-580 (2016).

4. Lin, Jia-Horng, Chien-Lin Huang, Chi-Fan Liu, Chih-Kuang Chen, Zheng-Ian Lin, and Ching-Wen Lou. Materials 8(12), 8279-8291(2015).

5. George Philip, Shantanu Bhowmik, Mathew Abraham, P. K. Sriram, Mohan kumar Pitchan, and G. Ajeesh. Proceedings of the Institution of Mechanical Engineers, Part L: Journal of Materials: Design and Applications 1464420716660874 (2016).

6. Mingliang G Demin, Journal of Reinforced Plastics and Composites. 28(1):5-16 (2008).

7. D. Pérez-Rocha, A. Morales-Cepeda, F. Navarro-Pardo, T. Lozano-Ramírez, P. LaFleur . International Journal of Polymer Science. 1-8 (2015).

8. A. Petrov Saakov A. Technology and equipment for plasma surface hardening of heavy-duty parts. Materials and Manufacturing Processes. 17(3):363-378 (2002).

9. N. Phong, M. Gabr, K. Okubo , B. Chuong, T. Fujii, Materials \& Design.,47:624$632(2013)$. 